\title{
TIPOGRAFIA PARA LEITURA EM TELAS DE DISPOSITIVOS DE INTERAÇÃO MÓVEL: a resolução dos suportes ainda constitui um problema para os designers?
}

\author{
Christopher Hammerschmidt \\ Universidade Federal do Paraná \\ chdeutschbr@yahoo.com.br \\ Carla Galvão Spinillo \\ Universidade Federal do Paraná \\ cgspin@gmail.com
}

\begin{abstract}
Resumo: Neste artigo, relata-se uma pesquisa que visou a verificar a atual qualidade das telas dos dispositivos de interação móvel (DIMs) disponíveis no mercado brasileiro. Este esforço se conduziu com o objetivo de avaliar os efeitos das limitações de resolução na tipografia destinada à leitura em meios digitais. Por meio de um referencial teórico específico sobre essa temática, define-se o problema como característica elementar da tipografia digital desde o início da adaptação da linguagem tipográfica aos equipamentos eletrônicos. Em seguida, descreve-se o levantamento de dados colhidos nos sites das cinco empresas responsáveis pelos maiores números de vendas de DIMs no Brasil. A partir de tais dados, caracterizam-se as limitações de resolução em telas de dispositivos móveis como uma questão ainda relevante para os designers atualmente.
\end{abstract}

Palavras-chave: tipografia, tecnologias digitais, dispositivos de interação móvel.

\begin{abstract}
This paper reports a study that investigated the current standard of screens in mobile interaction devices (MIDs) available in the Brazilian market. Precisely, such initiative seeks to assess the effects of limitations regarding screen resolution to the work of designers with typography for reading in digital media. Based on a bibliographic research about this subject, we define the problem concerned as an elementary feature of digital typography since it was first adapted to display in electronic devices. Following, the data collection brings information about quality of screens from a range of devices, as identified in the websites of the five manufacturers with higher sales of MIDs in Brazil. Relying on the results of this study, it is possible to characterise the limitations in resolution for displaying type on screens of mobile interaction devices as a still relevant issue for designers today.
\end{abstract}

Keywords: typography, digital technologies, mobile interaction devices. 


\section{INTRODUÇÃO}

A tipografia consiste num código gráfico que revela grande riqueza e alta complexidade. Trata-se de uma linguagem visível, relacionada tanto à criação quanto ao uso de símbolos ortográficos (letras) ou para-ortográficos (numerais, pontuação entre outros) com finalidade de reprodução (FARIAS, 2001, p. 15). Uma característica marcante da tipografia reside no forte caráter funcional que lhe é próprio: seu objetivo consiste em representar e fortalecer visualmente o conteúdo de uma determinada mensagem, conforme se constata pela assertiva de Bringhurst (2005, p. 23): "A tipografia existe para honrar seu conteúdo".

Tal integração entre os aspectos formais e práticos (funcionais) na linguagem tipográfica remete à importância das questões técnicas para o desenvolvimento dessa área. Segundo Rocha (2012, p. 13), "desde o início, a tipografia estabeleceu relações que, além dos aspectos estéticos e econômicos, priorizaram fundamentalmente as questões técnicas". Dessa forma, a evolução da tipografia, dos tipos móveis aos processos fotomecânicos e depois com as tecnologias digitais, apresentou muitos desafios para a adaptação da linguagem tipográfica a cada meio de produção e de reprodução dos tipos (GAUDÊNCIO JÚNIOR, 2004; LICKO, 1990a). Mesmo considerando os avanços tecnológicos implementados nos ambientes digitais, as questões relacionadas a tecnologias de exibição (resolução das telas), disponibilidade de tipos, variações de cor, contraste e tamanho continuam a ser problemáticas e os dispositivos móveis potencializam essas dificuldades (ZWICK; SCHMITZ; KÜHL, 2005, p. 118; HOOBER; BERKMAN, 2012, p. 491; NOKIA, 2012, não paginado).

A popularização dos dispositivos de interação móvel ${ }^{1}$ (DIMs) tem levado as vendas a superarem as de computadores pessoais no mundo (SCHELLER; FRAGA, 2012, não paginado). No Brasil, a disseminação de smartphones tem sido notável. Por meio de reduções de impostos para esses aparelhos, a consequente queda nos preços de tais produtos e a oferta de planos de internet pré-pagos, o número de acessos móveis a conteúdo online cresceu 54\% de 2012 para 2013 (MONTE, 2011; CANDIDO, 2012; TAGIAROLI, 2012; G1, 2013). Já com relação à venda dos aparelhos, verificou-se um crescimento de 147\% entre os terceiros trimestres de 2012 e 2013 (UOL, 2013, não paginado). Pela primeira vez, as vendas desse tipo de dispositivo superaram os números registrados para celulares comuns no Brasil (REUTERS, 2014, não paginado).

Diante desse cenário crescente de mercado, a tipografia digital se encontra diante de novos paradigmas. Compreender como os princípios do design de tipos e da composição tipográfica se aplicam à realidade desses dispositivos, tendo em vista os recursos e as limitações tecnológicas, mostra-se fundamental para que se possa aproveitar as potencialidades comunicativas que tais aparelhos oferecem aos usuários.

\section{TIPOGRAFIA PARA LEITURA EM TELAS}

A tipografia digital revelou aos designers desafios bastante peculiares em relação às tecnologias precedentes. Com a necessidade de adaptar os desenhos dos tipos à limitada malha de pixels das telas dos primeiros computadores e a baixa definição das

\footnotetext{
${ }^{1}$ Neste artigo, considera-se a definição de dispositivos de interação móvel conforme a caracterização proposta por Pottes (2012, p. 29-33). Assim, a categoria dos DIMs abrange os aparelhos smartphones e MP4 (e.g. iPod Touch). Computadores laptops e tablets não podem ser classificados como DIMs, conforme sustenta o autor.
} 
impressoras então existentes, muitos profissionais da área consideravam o meio digital impróprio para o desenvolvimento da tipografia, como relata Licko (1990b, não paginado, tradução nossa): "Desde que fui apresentada ao design gráfico, eu ouvia todos dizerem o quanto as fontes digitais eram ruins e como era impossível melhorálas." De modo similar, Zapf (1987, p. 11, tradução nossa) relata que os tipos digitais eram "difíceis de ler, alguns ignorando todas as regras básicas da legibilidade." $\mathrm{O}$ autor argumenta ainda que os problemas no design dos tipos se relacionavam a uma conjunção de fatores:

Não apenas o nível tecnológico da época impunha limites à criação de bons desenhos de letras, mas também o prazer pela experimentação às vezes se mostrava um fator predominante na criação de tipos usando meios eletrônicos." (ZAPF, 1987, p. 11, tradução nossa)

O desenvolvimento de técnicas e ferramentas adequadas permitiu que designers de tipos projetassem soluções específicas para a reprodução da tipografia em meios digitais. Isso demandou a criação de fontes tipográficas capazes de tirar proveito das características dos dispositivos então existentes, visto que a adaptação de tipos tradicionais usados em metal e em fotocomposição muitas vezes se revelava impossível pela limitação física dos aparelhos eletrônicos, sobretudo a baixa resolução das telas (LICKO, 1990b, não paginado; ZAPF, 1987, p. 12-13).

Na década de 1990, a disseminação de dispositivos como telefones celulares, videogames portáteis e PDAs (personal digital assistants), além da crescente produção de conteúdo para visualização diretamente em tela, reafirmou a relevância das fontes projetadas para meios digitais (LUPTON, 2013, p. 25). Prova disso se encontra no investimento de grandes companhias em projetos de fontes tipográficas para suportes eletrônicos nesse período. Segundo Carter (2002, p. 123, tradução nossa),

\footnotetext{
$\mathrm{O}$ argumento completo de uma fonte para tela reside no fato de que as telas são muito grosseiras quando comparadas a materiais impressos. A resolução espacial dos monitores mal se alterou em todo esse tempo. De acordo com a Microsoft, não era possível prever quando telas de alta resolução acessíveis estariam à nossa disposição - havia uma barreira física contra isso.
}

Benson, Olewiler e Broden (2005, p. 5) corroboram essa contraposição das telas a materiais impressos. De acordo com os autores, historicamente as limitações na resolução das telas impediram que se alcançasse em ambientes digitais o mesmo nível de refinamento tipográfico possível na impressão.

Avanços tecnológicos mais recentes permitiram o aumento da resolução de telas de DIMs, de modo que muitas destas já ostentam qualidade de definição consideravelmente mais alta que a resolução de monitores usados em computadores desktop (ZWICK; SCHMITZ; KÜHL, 2005, p. 119). Entretanto, Felici (2011, p. 286) considera que só é possível atingir altos padrões de qualidade para a tipografia em telas com resolução próxima a 300 pixels por polegada (ppi). A partir da comparação exposta na Figura 1, pode-se perceber claramente o efeito da resolução das telas na forma como os tipos são exibidos (BROWN, 2011, p. 66). 
A

originality

B

originality

originality

Figura 1 - Exemplos da fonte Verdana exibida em telas de alta e baixa resolução A: tela de alta resolução com suavização dos contornos. B: tela de baixa resolução com contornos suavizados. C: tela de baixa resolução sem suavização dos contornos. Nos três casos, a fonte foi composta em corpo 13 pixels.

Fonte: Brown, 2011, p. 66.

Hoober e Berkman (2012, p. 492) sustentam que, mesmo havendo telas de alta resolução disponíveis atualmente, as questões básicas relacionadas à exibição de tipos em meio digital ainda persistem e devem ser consideradas durante o processo de design e de seleção de fontes tipográficas para conteúdo a ser acessado em DIMs. Partindo dessa premissa, o presente artigo apresenta um estudo comparativo das resoluções de telas de DIMs dos cinco fabricantes com números mais expressivos de vendas no Brasil, objetivando responder à seguinte pergunta: a resolução dos suportes digitais ainda constitui um problema para os designers? Com isto, busca-se oferecer uma contribuição pertinente tanto a profissionais que projetam fontes para dispositivos móveis quanto a designers que fazem uso da tipografia na elaboração de conteúdo para acesso por meio desses aparelhos. Ao gerar dados que sintetizam as características das telas de DIMs no Brasil, pode-se confirmar ou negar a validade de recomendações para o design de materiais destinados à leitura em telas. Estas abrangem desde comentários sobre a inadequação do uso de fontes com detalhes muito delicados, serifas finas e linhas de espessura reduzida, até considerações sobre economia de espaço e proporções no desenho dos tipos, conforme se verifica nos trabalhos de autores como Zwick, Schmitz e Kühl (2005), Felici (2011), Hoober e Berkman (2012), entre outros.

\section{ANÁLISE COMPARATIVA DA RESOLUÇÃO DE TELAS DE DIMS VENDIDOS NO BRASIL}

\subsection{Amostra e procedimentos metodológicos}

Para definir as empresas a comporem a amostra analisada neste estudo, considerouse dados provenientes da Gartner Inc. (citados por GRIPA, 2014, não paginado), empresa líder em pesquisas na área de tecnologia de informação e consultoria, atuando nesse ramo desde 1979. Assim, foram identificados cinco fabricantes para compor este estudo, os quais detêm fatias de mercado que totalizam mais de $90 \%$ das vendas de DIMs no Brasil: Samsung (54,3\%), LG (21\%), Motorola (6,7\%), Apple (6,4\%) e Nokia (3,8\%). 
Por meio de consultas aos sites dessas empresas, fez-se um mapeamento dos modelos de smartphones ${ }^{2}$ disponíveis no mercado brasileiro. Buscou-se, nas listagens de produtos de cada fabricante, a categoria correspondente aos DIMs, para identificar os modelos disponíveis e suas especificações detalhadas. Uma vez que nem todas as empresas fornecem os dados referentes à resolução das telas dos dispositivos, esse atributo foi calculado com base em outros dados fornecidos pelos fabricantes. Utilizouse a seguinte fórmula para o cálculo da resolução de telas de DIMs:

$$
\text { Resolução (em ppi) }=\frac{\sqrt{\text { largura }^{2}(\text { em pixels })+\text { altura }^{2}(\text { em pixels })}}{\text { diagonal (em polegadas })}
$$

Dessa maneira, pôde-se estabelecer um inventário apropriado aos objetivos deste estudo com relação aos modelos de smartphones que se encontram atualmente à disposição dos consumidores brasileiros. Adotou-se como parâmetro para avaliação das resoluções de tela o valor de 300 ppi, considerado por Felici (2011, p. 286) o mínimo imprescindível para se obter uma qualidade satisfatória à exibição da tipografia em suportes digitais. No próximo item, exibem-se os resultados da pesquisa.

\subsection{Resultados e discussão}

O levantamento dos dados indicou a existência de 134 modelos de DIMs produzidos pelas cinco principais empresas desse ramo no mercado brasileiro. Os dados aferidos para resolução de tela demonstraram haver uma sensível variedade nas técnicas empregadas para a exibição de imagens nesses aparelhos. Portanto, para organizar os resultados e permitir discussões mais direcionadas neste artigo, optou-se por estabelecer categorias para a qualidade de definição das telas.

Assim, estas foram classificadas como telas de baixa, média ou alta resolução. A primeira classe abrange aquelas com densidade de pixels inferior a $150 \mathrm{ppi}$. O estrato das telas de alta resolução, por outro lado, compreende aquelas com 300 ppi ou mais. Os valores intermediários definem o que se denominou resolução média. Esta categoria se estrutura em três faixas: M1, de 150 a 199 ppi, valores mais próximos àqueles que caracterizam baixas resoluções; M2, de 200 a 249 ppi, valores médios; M3, de 250 a 299 ppi, valores mais próximos aos que definem altas resoluções. Os números gerais referentes às quantidades de modelos de DIMs incluídas em cada categoria compõem a Tabela 1.

Tabela 1 - Quantidade de modelos de DIMs em cada categoria de resolução de tela

\begin{tabular}{ccccccc}
\hline & \multicolumn{7}{c}{ CATEGORIAS DE RESOLUÇÃO DE TELAS } & \multirow{2}{*}{ Total } \\
\cline { 2 - 6 } & Baixa & M1 & M2 & M3 & Alta & 134 \\
\hline $\mathbf{n}$ & 24 & 33 & 36 & 16 & 25 & 100,00 \\
\hline$\%$
\end{tabular}

Fonte: Elaboração do autor, com base na pesquisa realizada.

\footnotetext{
${ }^{2}$ No caso da empresa Apple, considerou-se também o dispositivo iPod Touch para a investigação, uma vez que, segundo a definição de DIM aqui empregada, esse aparelho está dentro do escopo da pesquisa.
} 
Os dados contidos na Tabela 1 revelam que a categoria com maior número de modelos de DIMs consiste naquela que congrega os aparelhos com telas de resolução média. A soma dos valores em M1, M2 e M3 totaliza 85 modelos dos 134 identificados na pesquisa, correspondendo a $63,44 \%$ dos dispositivos. Já no estrato das telas de alta resolução, encontraram-se apenas 25 modelos de DIMs - quantidade equivalente a menos de $20 \%$ do total de modelos comercializados pelas cinco principais empresas que vendem tais aparelhos no Brasil.

Tomando-se isoladamente os resultados obtidos para cada fabricante, também se pode estabelecer considerações pertinentes. Constatou-se que a Samsung, além de ser responsável pelo maior número de vendas de smartphones no Brasil (GRIPA, 2014, não paginado), estabelece-se ainda como a empresa que oferece a maior variedade de modelos desses aparelhos no país. Ao todo, identificaram-se 60 modelos, ou seja, $44,78 \%$ dos 134 oferecidos pelas cinco empresas pesquisadas. Todavia, a proporção de dispositivos dotados de telas de alta resolução se revelou mais baixa que no geral: apenas 7 modelos se encaixam nessa definição, isto é, 11,67\% dos modelos vendidos por essa empresa. A Tabela 2 apresenta a distribuição das quantidades de modelos em cada categoria para DIMs fabricados e vendidos pela Samsung. As porcentagens dizem respeito ao número total de modelos dessa mesma marca.

Tabela 2 - Quantidade de modelos de DIMs fabricados e vendidos pela Samsung em cada categoria de resolução de tela

\begin{tabular}{ccccccc}
\hline & \multicolumn{7}{c}{ CATEGORIAS DE RESOLUÇÃo DE TELAS } & \multirow{2}{*}{ Total } \\
\cline { 2 - 6 } & Baixa & M1 & M2 & M3 & Alta & 7 \\
\hline $\mathbf{n}$ & 12 & 14 & 18 & 9 & 70 & 60 \\
\hline$\%$ & 20,00 & 23,33 & 30,00 & 15,00 & 11,67 & 100,00 \\
\hline
\end{tabular}

Fonte: Elaboração do autor, com base na pesquisa realizada.

Em segundo lugar na quantidade de aparelhos vendidos, a LG também fica atrás apenas da Samsung no que diz respeito ao número de modelos disponibilizados aos consumidores brasileiros. Dos 29 tipos de smartphones oferecidos pela LG (21,64\% dos 134 totais), verificou-se que apenas 6 se enquadram na categoria de telas de alta resolução, ou seja, 20,69\% dos exemplares dessa empresa. Na Tabela 3, encontra-se a relação completa de dados referentes à distribuição de modelos nas categorias de resolução de telas para DIMs produzidos pela LG.

Tabela 3 - Quantidade de modelos de DIMs fabricados e vendidos pela LG em cada categoria de resolução de tela

\begin{tabular}{ccccccc}
\hline & \multicolumn{7}{c}{ CATEGORIAS DE RESOLUÇÃO DE TELAS } & \multirow{2}{*}{ Total } \\
\cline { 2 - 6 } & Baixa & M1 & M2 & M3 & Alta & 29 \\
\hline $\mathbf{n}$ & 9 & 4 & 9 & 1 & 6 & 20,69 \\
\hline$\%$ & 31,03 & 13,79 & 31,03 & 3,45 & 20,00 \\
\hline
\end{tabular}

Fonte: Elaboração do autor, com base na pesquisa realizada. 
A quantidade de modelos fabricados pela Motorola se revelou consideravelmente mais baixa que as das empresas anteriormente mencionadas. Entre os 134 modelos de DIMs encontrados na pesquisa, somente 16 remetiam à Motorola, o que corresponde a $11,94 \%$ do total. Quanto à distribuição de exemplares nos estratos que definem a qualidade de resolução de tela, verificou-se a ausência de dispositivos classificados como baixa resolução. A soma das quantidades incluídas nas categorias intermediárias revela ser esta a classe mais numerosa entre os DIMs da fabricante em questão: 13 modelos, $81,25 \%$ daqueles produzidos pela empresa. Os aparelhos com telas de alta resolução computaram apenas 3 modelos, sendo $18,75 \%$ dos smartphones da companhia. $\mathrm{O}$ detalhamento desses dados se encontra na Tabela 4.

Tabela 4 - Quantidade de modelos de DIMs fabricados e vendidos pela Motorola em cada categoria de resolução de tela

\begin{tabular}{lcccccc}
\hline & \multicolumn{7}{c}{ CATEGORIAS DE RESOLUÇÃo DE TELAS } & \multirow{2}{*}{ Total } \\
\cline { 2 - 6 } & Baixa & M1 & M2 & M3 & Alta & 16 \\
\hline $\mathbf{n}$ & - & 7 & 2 & 4 & 3 & 100,00 \\
\hline$\%$
\end{tabular}

Fonte: Elaboração do autor, com base na pesquisa realizada.

Os resultados obtidos para a análise de DIMs fabricados pela Apple revelou algumas particularidades em relação às demais empresas pesquisadas. Primeiramente, destacase a pequena variedade de modelos desses aparelhos, somando apenas 5 exemplares. Estes também se evidenciam por possuir telas de alta resolução, o que faz da Apple a única empresa a não oferecer mais dispositivos de baixa ou média resolução na categoria dos DIMs, como evidencia a Tabela 5. A análise de dispositivos dessa empresa não considerou unicamente os smartphones (diferentes modelos de iPhone), e incluiu os modelos de iPod Touch (MP4) disponibilizados por esse fabricante.

Tabela 5 - Quantidade de modelos de DIMs fabricados e vendidos pela Apple em cada categoria de resolução de tela

\begin{tabular}{|c|c|c|c|c|c|c|}
\hline & \multicolumn{5}{|c|}{ CATEGORIAS DE RESOLUÇÃO DE TELAS } & \multirow[b]{2}{*}{ Total } \\
\hline & Baixa & M1 & M2 & M3 & Alta & \\
\hline$n$ & - & - & - & - & 5 & 5 \\
\hline$\%$ & - & - & - & - & 100,00 & 100,00 \\
\hline
\end{tabular}

Fonte: Elaboração do autor, com base na pesquisa realizada.

Por fim, analisaram-se ainda os smartphones produzidos pela Nokia, a quinta maior empresa do ramo em número de vendas desses aparelhos no Brasil. Conquanto se trate do fabricante detentor da menor fatia do mercado brasileiro entre aqueles contemplados na pesquisa, a Nokia se destaca por ter a terceira maior variedade de modelos, atrás somente de Samsung e LG. Dos 134 modelos identificados no geral, verificou-se que 24 são fabricados pela Nokia. Seguindo a tendência apontada pelas outras empresas, à exceção da Apple, a maioria dos modelos ostenta telas de resolução intermediária. Esses aparelhos somam 17 exemplares (70,83\%) entre os dispositivos da 
mesma empresa. Já no que diz respeito aos smartphones com telas de alta resolução, nota-se que estes representam $16,67 \%$ dos DIMs desse fabricante, estando disponíveis apenas 4 modelos, conforme demonstra a Tabela 6 .

Tabela 6 - Quantidade de modelos de DIMs fabricados e vendidos pela Nokia em cada categoria de resolução de tela

\begin{tabular}{ccccccc}
\hline & \multicolumn{7}{c}{ CATEGORIAS DE RESOLUÇÃO DE TELAS } & \multirow{2}{*}{ Total } \\
\cline { 2 - 6 } & Baixa & M1 & M2 & M3 & Alta & 24 \\
\hline $\mathbf{n}$ & 3 & 8 & 7 & 2 & 4 & 100,00 \\
\hline$\%$
\end{tabular}

Fonte: Elaboração do autor, com base na pesquisa realizada.

O levantamento de dados referentes à resolução de telas de DIMs revelou a predominância de aparelhos com definição intermediária, ainda que a tecnologia para a produção de telas com maior densidade de pixels já se encontre disponível. Isso parece indicar um movimento no sentido do aumento da resolução das telas dos dispositivos, de modo a possibilitar o desenvolvimento de uma tipografia mais refinada em meios digitais (FELICl, 2011). Mesmo os aparelhos com menor qualidade de exibição analisados nesta pesquisa mostraram resoluções superiores àquelas que comumente caracterizam monitores de computadores desktops, confirmando as afirmações de Zwick, Schmitz e Kühl (2005).

Os resultados obtidos ao longo da pesquisa descrita neste artigo permitem que se estabeleçam algumas conclusões e considerações finais acerca do tema desenvolvido.

\section{CONCLUSÃO E CONSIDERAÇOES FINAIS}

Os resultados da análise comparativa entre as telas dos DIMs das cinco principais empresas atuantes no mercado brasileiro levam a concluir, em resposta à pergunta de pesquisa, que as resoluções dos suportes digitais ainda constituem um problema que deve ser considerado pelos designers. $O$ caráter afirmativo deste estudo corrobora para a importância de conhecer as limitações dos meios digitais no que tange à reprodução da linguagem tipográfica, conforme apontado nas recomendações constantes da literatura (ZWICK; SCHMITZ; KÜHL, 2005; FELICI, 2011; HOOBER; BERKMAN, 2012).

Considerando a trajetória da tipografia digital, vale comentar que as questões relacionadas à resolução das telas se mostraram problemáticas desde os primórdios do desenvolvimento da tipografia digital. A dificuldade de adaptar as formas tradicionais do design de fontes originalmente projetadas para a composição de tipos móveis à grosseira malha de pixels das primeiras telas de baixa resolução impôs desafios a engenheiros e designers. Por um lado, a resolução de problemas envolveu a elaboração de tipos específicos para os ambientes digitais caracterizados pela baixa densidade de pixels para exibição de conteúdo. Por outro, o aumento nas resoluções das telas dos dispositivos eletrônicos tende a tornar essas considerações cada vez menos relevantes, permitindo que os tipos para visualização em telas se aproximem daqueles destinados à confecção de materiais impressos. Os resultados deste estudo apontam que estas questões ainda possuem relevância no contexto atual dos dispositivos de interação móvel, visto que podem limitar alguns recursos de articulação tipográfica e influenciar a 
seleção de tipos por parte dos designers, como o uso de desenhos dotados de detalhes muito finos, serifas frágeis e linhas de espessura tênue, que tendem a desaparecer em suportes de exibição com baixas ou médias densidades de pixels.

Nesse sentido, tanto os designers que projetam tipos para a leitura em meios digitais quanto aqueles que desenvolvem layouts e precisam escolher fontes tipográficas precisam estar cientes das limitações físicas de grande parte dos aparelhos presentes no mercado. A elaboração de soluções adequadas aos dispositivos por meio dos quais os usuários terão acesso ao conteúdo exige um equilíbrio entre o refinamento permitido pelos aparelhos mais desenvolvidos e a qualidade de exibição ainda insuficiente das telas de uma fatia considerável do mercado de DIMs no Brasil. Embora os dados oferecidos por este estudo sugiram que a disseminação de aparelhos com telas de maior densidade de pixels já constitui realidade, não se pode prever em quanto tempo as limitações da malha de pixels das telas deixarão de impor restrições ao trabalho dos designers.

Portanto, considera-se que pesquisas como esta devem ser realizadas com certa frequência, estabelecendo um mapeamento dos dispositivos aos quais as pessoas têm acesso. Também se julga pertinente que investigações deste tipo não se restrinjam à simples análise de dados disponíveis pelos fabricantes, mas que sejam executados estudos diretamente com os usuários de DIMs. Dessa maneira, pode-se obter um levantamento ainda mais confiável e próximo da realidade do contexto dos usuários.

\section{REFERÊNCIAS}

BENSON, Jared; OLEWILER, Ken; BRODEN, Nancy. Typography for mobile phone devices: the design of the QUALCOMM sans font family. In: Conference on Designing for User eXperience, 2., 2005, San Francisco. Proceedings... New York: AIGA, 2005, p. 2-18.

BRINGHURST, Robert. Elementos do estilo tipográfico: versão 3.0. Tradução de André Stolarski. São Paulo: Cosac Naify, 2005.

BROWN, Tim. Type rendering \& web fonts. 8 Faces, n. 3, p. 64-67, 2011.

CANDIDO, Fabiano. Smartphone ficará $10 \%$ mais barato em outubro, diz ministro. INFO Online, 12 out. 2012. Seção Mercado. Disponível em: <http://info.abril.com.br/noticias/ mercado/smartphone-ficara-10-mais-barato-em-outubro-diz-ministro-08102012-31.shl>. Acesso em: 20 out. 2012.

CARTER, Matthew. Matthew Carter. In: EARLS, David. Designing typefaces. Mies: RotoVision, 2002.

FARIAS, Priscila Lena. Tipografia digital: o impacto das novas tecnologias. 3. ed. Rio de Janeiro: 2AB, 2001.

FELICl, James. The complete manual of typography: a guide to setting perfect type. 2. ed. Berkeley, CA: Adobe, 2011.

G1. Conexões à internet rápida móvel crescem 54\% em março no Brasil. 28 mar. 2013. Seção Tecnologia e Games. Disponível em: <http://g1.globo.com/tecnologia/noticia/ 2013/03/conexoes-internet-rapida-movel-crescem-54-em-marco-nobrasil.html>.

Acesso em: 2 maio 2013.

GAUDÊNCIO JÚNIOR, Norberto. A herança escultórica da tipografia. São Paulo: Rosari, 2004. 
GRIPA, Marcelo. Samsung detém mais da metade do mercado de smartphones no Brasil. Olhar digital, 12 fev. 2014. Seção Últimas notícias. Disponível em: <http:// olhardigital.uol.com.br/pro/noticia/samsung-detem-mais-da-metade-do-mercado-desmartphones-no-brasil/40282>. Acesso em 5 abr. 2014.

HOOBER, Steven; BERKMAN, Eric. Designing mobile interfaces. Sebastopol, CA: O’Reilly, 2012.

LICKO, Zuzana. Typeface design: Zuzana Licko, 1990a. In: Vanderlans, R. (Org.) Emigre n. 70 the look back issue: selections from Emigre Magazine 1-69. Celebrating 25 years of graphic design. Berkeley, CA: Gingko Press, 2009. p. 89-92.

LICKO, Zuzana. Interview with Zuzana Licko. 1990b. Emigre Fonts. Disponível em: <http:// www.emigre.com/Licko3.php>. Acesso em 15 mar. 2014. Entrevista concedida a Rudy VanderLans. Publicada originalmente em Emigre, n. 15, 1990.

LUPTON, Ellen. Pensar com tipos: guia para designers, escritores, editores e estudantes. 2. ed. São Paulo: Cosac Naify, 2013.

MONTE, Fabiana. Governo quer levar benefícios fiscais para smartphones. Brasil Econômico, 30 nov. 2011. Seção Telecomunicações. Disponível em: <http://www. brasileconomico.com.br/noticias/governo-quer-levar-beneficios-fiscais-para-smartphones_ 109892.html>. Acesso em: 20 out. 2012.

NOKIA Developer Wiki. Typography in mobile devices, 2012. Disponível em: <http:// www.developer.nokia.com/Community/Wiki/Typography_in_Mobile_Devices>. Acesso em: 15 mar 2014.

REUTERS. Vendas de smartphones no Brasil mais que dobram em 2013. INFO Online. 2 abr. 2014. Seção Mercado. Disponível em: <http://info.abril.com.br/noticias/mercado/ 2014/04/vendas-de-smartphones-no-brasil-mais-que-dobram-em-2013.shtml>. Acesso em: 5 abr. 2014.

ROCHA, Claudio. Novo projeto tipográfico: análise e produção de fontes digitais. São Paulo: Rosari, 2012.

SCHELLER, Fernando; FRAGA, Nayara. Vendas de smartphones têm forte alta e já superam as de PCs no mundo. O Estado de S. Paulo, 6 fev. 2012. Seção Economia \& Negócios. Disponível em: <http://economia.estadao.com.br/noticias/economia,vendasde-smartphones-tem-forte-alta-e-ja-superam-as-de-pcs-no-mundo,102001,0.htm>.

Acesso em: 16 jul. 2012.

TAGIAROLI, Guilherme. Planos baratos e pré-pagos visam popularizar internet móvel; veja quanto custa o acesso. UOL Notícias, 21 mar. 2012. Seção Tecnologia. Disponível em: <http://tecnologia.uol.com.br/ultimas-noticias/redacao/2012/03/21/operadorasapostam-em-internet-movel-barata-e-pre-para-popularizar-acessos.jhtm>. Acesso em: 22 abr. 2013.

ZAPF, Hermann. Preface. In: KAROW, Peter. Digital formats for typefaces. Hamburg: URW, 1987. p. 11-14.

ZWICK, Carola; SCHMITZ, Burkhard; KÜHL, Kerstin. Designing for small screens. Lausanne: AVA, 2005. 